\title{
La empresa como ente relacional. Una respuesta intermedia a la cuestión de su estatus ontológico
}

\author{
MARÍA MARTA PREZIOSA ${ }^{1}$ \\ Pontificia Universidad Católica Argentina (Argentina) \\ mmpreziosa@uca.edu.ar
}

\section{Resumen}

En este trabajo se ofrece una respuesta a la cuestión del estatus ontológico de la empresa basada en la noción de relación de la filosofía aristotélico-tomista. Para ello se analizan argumentos individualistas y colectivistas, y argumentos de autores que proponen superar esa antinomia mediante la noción de relacionalidad. Dado que dichos autores se quedan en el plano fenoménico, se ofrece un análisis que provee un adecuado fundamento metafísico a las interacciones que se dan entre los integrantes de la empresa y conforman una realidad distinta a ellos, pero que inhiere en ellos. La empresa se presenta como una realidad relacional y sinérgica ordenada por una estructura, una cultura y líderes, que modifica a sus miembros en habilidades, capacidades y hábitos compartidos.

Palabras clave: estatus ontológico de la empresa, agencia moral de la empresa, filosofía aristotélico-tomista, relación real, relación de razón, ente relacional.

\section{The Company as a Relational Entity. An Intermediate Position on Corporate Ontological Status}

\begin{abstract}
This paper offers an answer to the ontological question based on the notion of relationship from Aristotelian-Thomistic philosophy. To this end, individualist and collectivist arguments are analyzed, as well as arguments by authors who propose to overcome this antinomy by means of the notion of relationality. Since these authors stop at the phenomenic level, this paper offers an analysis that provides an adequate metaphysical foundation to interactions. These real relationships modify its subjects in an accidental way composing a different reality, but not a substantial reality. The company is presented as a relational and synergic reality ordered by a structure, a culture and leaders that modifies its members in shared skills, capabilities and habits.
\end{abstract}

Key words: ontological status of the corporation, corporate moral agency, aristotelian-thomist philosophy, real relation, relation of reason, relational being.

1 Doctora en Filosofía, Universidad de Navarra (2015). Máster en Administración de Empresas, IDEA, Escuela Superior de Administración (1992). Profesora Titular de Ética de los Negocios. Directora de la Certificación en Compliance, ética y derecho (UCA). Entre sus publicaciones recientes se cuenta el libro La mentalidad compartida en la empresa (2016). Y los artículos "El entrenamiento ético en un programa de integridad" (2018); "Cómo los empleados simbolizan el poder, los logros y la empresa. Etnografía de una filial latinoamericana de una empresa multinacional" (2017); "Imágenes de la vida organizacional" (2016). 


\section{INTRODUCCIÓN}

Se ha dicho que el siglo XX fue el siglo de las grandes organizaciones. Sean las instituciones de los estados organizados o las empresas y corporaciones, éstas crecieron y adquirieron un enorme poder. Las empresas no solo cobijaron empleos, sino que mediante la producción a gran escala abarataron e hicieron accesibles muchos productos; no solo ofrecieron nuevos servicios, sino que también generaron nuevos daños, más potentes en su dimensión y alcance. Las corporaciones han protagonizado también ya entrado nuestro siglo diversos tipos de escándalos: financieros de impacto sistémico, ambientales, de participación en delitos de corrupción, así como también de colaboración con guerras o regímenes totalitarios.

Hoy, las grandes organizaciones nos siguen ofreciendo un ámbito para la reflexión moral. Un espacio donde el individuo actúa junto con otros de modo organizado, cumpliendo roles y funciones. Un lugar donde muchas profesiones, que antes se ejercían de forma liberal, se realizan en y a través de una o varias organizaciones. Este ámbito se caracteriza por cierto tipo de influencias específicas sobre la moralidad del individuo, tales como las presiones de pares y superiores o las mediciones de desempeño y recompensas.

Dada la influencia que ejerce la organización en el individuo y el mayor impacto que tienen las organizaciones, surge la pregunta acerca de si es posible considerar a la empresa moralmente responsable. Es decir, además de la responsabilidad de sus directivos ¿es posible atribuir una responsabilidad moral a la organización en sí?; si es posible, entonces ¿es la empresa un ente distinto de sus integrantes?

\subsection{La perspectiva legal}

En el mundo económico angloamericano, la preocupación social por el rol de las grandes empresas se ha ido reflejando en diversos debates legales que afectan el mundo corporativo. De acuerdo con el historiador de instituciones económicas Richard P. Adelstein (2013), a partir del año 1870 , las leyes angloamericanas comenzaron a considerar a las corporaciones bajo una óptica colectivista. Es decir, ya no como una mera ficción jurídica, según la visión individualista propia del common law y del liberalismo lockeano. Los principales referentes del colectivismo fueron, según Adelstein, el británico Frederic W. Maitland, historiador y abogado (18501906) y el abogado norteamericano Ernst Freund (1864-1932). Esta visión de la corporación se ha mantenido en el tiempo, según afirma el eticista británico Geoff Moore (1999) y se evidencia en que las leyes corporativas en EE. UU. y en el Reino Unido están, de hecho, construidas sobre la base 
de que las empresas son moralmente responsables. El argumento es: aunque no se pueda identificar dentro de la empresa la mente directora que ha conducido a la comisión del delito, es observable que la empresa produce más daño social que las personas físicas aisladas o que actúan por su cuenta (Moore, 1999).

Como señalan Walt \& Schwartzman (2017) y Moore (1999), el hecho de que la ley reconozca la responsabilidad de la empresa como tal, no es fundamento suficiente para afirmar que este reconocimiento sea moralmente correcto. Sin embargo, es importante destacar la tendencia legal corporativa que cada vez más atribuye responsabilidad a la empresa en sí, más allá de sus individuos o sus directivos. Un ejemplo claro de esta tendencia, según el especialista en ética organizacional norteamericano Kevin W. Gibson (2011) son las Federal Sentences Guidelines for Organizations de EE. UU. ${ }^{2}$ que "cambian conceptualmente la aproximación al delito corporativo" reconociendo la influencia del entorno organizacional en el individuo y promoviendo la implementación de sistemas de Compliance ${ }^{3}$. Para el británico Moore (1999) un ejemplo de esta tendencia es a ley inglesa sobre Corporate Manslaughter. Según esta norma, una empresa puede ser considerada homicida en sentido culposo, es decir, la empresa puede ser encontrada responsable de una muerte no intencionada, como es en el caso de accidentes mortales provocados por defectos de fabricación de un producto $^{4}$ o por la falta de mantenimiento en un sistema público de transporte.

En Latinoamérica, esta tendencia se plasma hoy en las leyes de responsabilidad de las personas jurídicas por delitos de corrupción, donde además de las sanciones penales a los directivos se prevén sanciones penales para la empresa en sí. En la República Argentina, en los considerandos del proyecto de la Ley 27.401 (2017) se señala que la sanción penal a la empresa se puede fundar, entre otros factores, en el haber sostenido algunos "defectos organizativos". Es decir, la empresa sería considerada penalmente responsable por no haber supervisado los procesos o a sus empleados, o bien por no haber alineado los incentivos comerciales a una cultura de integridad facilitando, de este modo, la comisión de un soborno a un funcionario público.

De fines de los años 80.

Compliance es un sistema de prevención de prácticas no éticas o delictivas en el entorno corporativo que suele consistir en un código de ética, entrenamiento y capacitación, canal de denuncias anónimo, investigaciones internas y sanciones.

4 El caso Ford Pinto, de EE. UU., en los años 70 es paradigmático de esta problemática. La ley mencionada es del 2008. 
Gibson (2011) subraya que las leyes están reconociendo el fenómeno que largamente ha estudiado la ética empresarial, a saber, la influencia recíproca y dinámica entre la organización y el empleado ${ }^{5}$.

1.2. La cuestión del estatus moral de la empresa en la disciplina ética empresarial

El debate sobre el estatus moral de la empresa o agencia moral de la empresa (a partir de ahora, $\mathrm{AME}^{6}$ ) se ha dado de modo profuso y extenso en el ámbito de la disciplina ética empresarial angloamericana. Para Moore (1999), este debate es un tema crucial, pero a menudo se deja de lado y sin resolver porque es un tema inherentemente complejo. Adelstein (2013), por su parte, también destaca su dificultad, señalando que ha desvelado a filósofos morales y filósofos del derecho por mucho tiempo.

A modo de síntesis se presentan algunas de las respuestas a la cuestión AME que se encuentran en la literatura angloamericana. Las mismas se podrían categorizar según su intensidad. En el extremo inicial de un hipotético vector se encontraría la negación de que la empresa sea pasible de atribución de responsabilidad moral. El extremo opuesto, el de más alta intensidad, corresponde a la afirmación de que la empresa es moralmente responsable como lo es una persona individual o incluso más que ella. El concepto de agente moral se puede considerar de intensidad media. La noción de agente moral es más limitada que la de persona, sin embargo, permite igualmente atribuir faltas morales a la empresa (Moore, 1999: 332).

En la dirección y sentido de este vector se ubicarían respuestas como las que siguen. La empresa no es moralmente responsable (McMahon, 1995). La empresa tiene relevancia moral, pero no tiene estatus moral (Heersmink, 2017). La empresa es causa de sus acciones colectivas, pero no tiene intencionalidad colectiva. Solo los individuos tienen intencionalidad (Velasquez, 2003). La empresa tiene racionalidad, toma decisiones y tiene capacidad de controlar sus actos, revisar sus estructuras y de buscar el consenso de las partes afectadas en las decisiones (Thomas Donaldson citado en Blair, 2016; Gibson, 2011). La empresa es sujeto de derechos y obligaciones. La empresa es un agente moral (Moore, 1999). La empresa puede tener intencionalidad delictiva (Phillips, 1995). La empresa tiene identidad, duración y capacidad de aprendizaje. La empresa tiene sociabilidad, es un miembro de la comunidad moral y puede reconocer las normas

5 En su definición de lo que es la disciplina ética empresarial, el filósofo Georges Enderle (1993) señala que uno de sus ejes centrales es la interacción entre el micro-level (individuo) y el meso-level (organización). El macro-level es el de la sociedad.

6 En inglés Corporate Moral Agency. 
del entorno y rendir cuentas frente a otros. La empresa tiene conciencia moral (Goodpaster \& Matthews, 1982; Goodpaster, 2007). La empresa es una persona moral (French, 1979). La empresa puede tener sensibilidad y sentimientos morales, puede estar atenta a las consecuencias de sus actos (French, 1979; Scruton en Adelstein, 2013). La empresa es un agente más pleno que una persona debido a su poder y tamaño (Mc Donald en Adelstein, 2013).

\subsection{La cuestión del estatus ontológico de la empresa}

La cuestión AME y la del estatus ontológico de la empresa (a partir de ahora, EOE) se encuentran de modo imbricado en la literatura, lo que abona a su dificultad. Específicamente, la cuestión EOE apunta a la naturaleza del ente empresa, pero ambas se suelen presentar entrelazadas debido a que se han considerado interdependientes. La cuestión EOE podría formularse del siguiente modo: ¿existe algo que es propio de la empresa y no reductible al individuo?; la empresa ¿tiene una realidad distinta de la de sus integrantes?; la empresa ¿es un ente real?; si es un ente distinto y/o real ¿qué tipo de realidad tiene?

Este escrito tiene como objetivo proveer argumentos de la filosofía aristotélico-tomista para sustentar una nueva respuesta a la cuestión del estatus ontológico que sea superadora de la antinomia individualismo-colectivismo y que recoja algunos de los últimos aportes al debate. El recurso a esta tradición de pensamiento no se ha encontrado en el debate anglosajón y permite zanjar algunas de las dificultades de la discusión.

Para cumplir con este objetivo, en primer lugar, se presentan dos autores importantes en el contexto del debate AME, el primero con argumentos colectivistas que sustentan que la empresa es una persona o un agente moral, y el segundo con argumentos individualistas que niegan que la empresa sea un ente distinto de sus integrantes (epígrafes 2-3). A continuación, se presentan los argumentos de dos autores representativos de quienes buscan una respuesta intermedia a la cuestión AME y que centran su análisis en el fenómeno de la relacionalidad en la empresa (epígrafe 4). Por último, se ofrecen las proposiciones aristotélico-tomistas en diálogo con los autores citados. Se sostiene que la empresa ${ }^{7}$ es un ente real distinto de sus integrantes sobre la base de una teoría metafísica de la relación (epígrafes 5-8).

En este trabajo nos limitaremos a llamar "empresa" al conjunto de múltiples formas que ésta puede adquirir, tanto desde el punto de vista jurídico como desde el punto de vista económico y operacional. 


\section{LO COLECTIVO O LO INDIVIDUAL}

Según McMahon (1995) el debate consiste en: o bien no existen los hechos sociales, o bien existen de modo no reductible al individuo. Para Moore (1999), la disputa es, o bien la empresa es un mero agregado de individuos, o bien es una persona moral plenamente desarrollada. Para Soares (2003), en cambio, las respuestas al debate son o bien nominalistas, o bien realistas. Para la respuesta metafísica nominalista, las corporaciones son colecciones o agregados de seres humanos, para la respuesta metafísica realista, la corporación "tiene una existencia y un significado tal como el de una personalidad moral/legal propia" (Soares, 2003: 144). En líneas generales, es posible afirmar que ambas cuestiones (AME y EOE) se han respondido o bien subrayando el peso ontológico del individuo, o bien enfatizando el peso ontológico de la empresa.

\subsection{Peter A. French}

French es un filósofo moral que actualmente se despeña en la Arizona State University, EE. UU. y es el iniciador y principal proponente de la cuestión AME. En su llamado seminal paper (French, 1979) intenta explicar distintos escándalos corporativos que no se podían explicar simplemente desde el punto de vista de las decisiones individuales de los ejecutivos (Gibson, 2011: 72).

French acuñó el concepto de estructura corporativa de decisión interna ${ }^{8}$ (ECDI, de ahora en adelante). La ECDI tiene dos elementos: una descripción clara (i.e. un diagrama de flujo organizacional) que indica cómo se han de tomar decisiones dentro de la estructura de poder (i.e. roles, puestos, niveles y responsabilidades) para llevar a cabo los objetivos de la firma y un conjunto de reglas que permiten el reconocimiento de las decisiones corporativas. Mediante esta estructura, una decisión individual se convierte en una acción colectiva, y de este modo, tanto los integrantes de la empresa, como los de fuera interpretan que las decisiones de los individuos representan las decisiones y acciones de la firma. Cuando la ECDI funciona, la persona actúa por las razones de la firma, por tanto, French afirma que se consigue una síntesis del acto individual en un acto de la empresa. Así, la ECDI le confiere a la empresa una forma particular de

\footnotetext{
${ }^{8}$ En ingles, Corporate Internal Decision Structure, CIDS.
} 
intencionalidad y agencia racional suficiente como para considerarla persona moral (moral person) y cargar con responsabilidades por los daños realizados con sus acciones colectivas?.

French (1984) también propuso una forma de determinar si una empresa es moralmente responsable mediante la formulación del "principio de enmienda reactiva" (Principle of Responsive Adjustment). El principio formula que si una empresa genera un daño involuntario (por ejemplo, un accidente de aviación o un choque de trenes) y no corrige su forma de operar luego de ocurrida la tragedia, la organización puede ser considerada moralmente responsable por consentir la continuidad de ese modo de operar. Es decir, si una empresa es sensible, va a adoptar cursos de acción correctivos en su ECDI para evitar que una tragedia vuelva a suceder.

Siguiendo a los comentadores de French, cabe afirmar: (a) la ECDI implica que queda un resto o residuo que debe ser atribuido a la empresa como tal, más allá de las responsabilidades individuales (Adelstein, 2013); (b) la ECDI es lo que le otorga carácter moral a la empresa y a su vez, lo revela (Gibson, 2011); (c) la ECDI, además, permite la atribución de intencionalidad delictiva corporativa (composite mens rea) (Phillips, 1995).

\subsection{Manuel G. Velasquez}

Velasquez es profesor de ética empresarial en Santa Clara University, EEEU. Sus argumentos se enmarcan en el individualismo metodológico, según su propia definición. Siguiendo el occamiano, Velasquez (2003) afirma que es más simple explicar la empresa solo por el individuo. Si bien reconoce que la coincidencia de objetivos entre los individuos que se coordinan para producir algo resulta en una acción colectiva, afirma que ello no implica que la empresa sea un ente real. Por ejemplo, una sinfonía es una acción colectiva, pero no es un organismo supraindividual.

Velasquez (2003) responde detalladamente a los colectivistas con argumentos metafísicos y legales. Considera que el hecho de que la gente se involucre en las organizaciones en comportamientos que nunca hubiese realizado de modo aislado, no debe llevar a reificar ${ }^{10}$ el grupo, ni las interacciones de sus miembros. Para Velasquez (2003), las presiones de la organización sobre el individuo son solo "metáforas mecánicas" y no hay

\footnotetext{
9 Según Moore (1999: 332) French luego cambió persona moral por el concepto de actor moral. Moore afirma que es mejor referirse a la empresa como agente moral y no como actor moral porque agente está más extendido en su uso.

10 No contamos con el verbo reificar en castellano. Sí existe en inglés to reify, hacer real y proviene del latín re/res: cosa.
} 
que considerarlas como si constituyesen un "espíritu organizacional fantasmagórico". Los individuos solo actúan por sus deseos y creencias. Por otra parte, afirma que la responsabilidad, entendida como la atribución de una acción y de sus consecuencias a un sujeto, se define por la intencionalidad, algo que la empresa no puede tener. Para Velasquez, por tanto, ni existe la mente de grupo, ni hay intencionalidad grupal, ni responsabilidad moral de la empresa, solo la responsabilidad de los individuos.

Velasquez igualmente reconoce que la empresa puede colectivamente ser causa de, por ejemplo, daños ambientales. Pero ser causalmente responsable no es ser moralmente responsable. En ese caso, debe pagar de forma compensatoria por los daños (liability). Velasquez considera que es peligroso afirmar la responsabilidad moral de la empresa en sí, no solo porque permite esconder las responsabilidades individuales, sino porque de ese modo se considera a las personas una mera parte de un todo. A Velasquez le preocupa que la responsabilidad individual se esconda tras el velo corporativo, por eso, niega la realidad de la empresa como ente distinto de sus integrantes.

\section{LO ONTOLÓGICO Y LO MORAL}

Como se mencionó, el estatus moral y ontológico de la empresa se han debatido de modo entrelazado. Según el filósofo social Christopher McMahon (1995), se ha considerado por mucho tiempo que la resolución de ambas cuestiones era necesariamente interdependiente. Moore (1999) también señala esta interdependencia.

En los últimos años, sin embargo, se han podido leer algunos análisis que intentan independizar la dimensión ontológica de la moral y así superar algunos de los aspectos del debate que quedan sin resolver. Por ejemplo, Buddeberg \& Hecker (2018) buscan una superación de la antinomia colectivismo-individualismo, en el marco teórico de la ética del discurso. Por otra parte, Walt \& Schwartzman (2017) aseveran que la disputa entre "ente real" y "ente agregado o ente de ficción" es un debate noconcluyente. "Los debates perennes sobre la ontología corporativa no solo no sorprenden, sino que son confusos tal como era de esperar" (2017:28). En la misma línea, Blair (2015) postula que ya no es importante si la corporación es un ente artificial o real o un agregado de personas, sino el razonamiento moral y la búsqueda de consensos. A su vez, Gibson señala que, si bien es incuestionable que la empresa tiene un estatus metafísico diferente a sus integrantes, el debate acerca de "si la corporación tiene suficientes cualidades para ser una persona" es distractivo y enmascara lo esencial (2011: 72). 
La independencia que se abre paso entre el análisis de la cuestión moral y la ontológica pareciera mutar las posiciones iniciales (individualismo vs. alguna forma de colectivismo) en dos variaciones o recreaciones de estas. Por un lado, la variación semántica como continuación del individualismo metodológico y el nominalismo y, por otro lado, la variación relacional, como continuación de algunas de las formas de colectivismo.

El claudicar de la dimensión ontológica del debate se podría explicar por varios factores. En primer lugar, si bien el debate es teorético, para que tenga relevancia tiene que tener un correlato en la realidad empresarial empírica. La realidad empírica de la empresa es sumamente compleja debido a sus diferentes formas jurídicas, económicas y organizativas. En segundo lugar, las leyes no siempre están fundadas en cuestiones ontológicas, sino en aspectos pragmáticos, hecho que también desafía la relevancia del debate. Por último, se podría decir también que el pensamiento metafísico ha caído en desuso y aún más como fundamento de lo ético. Es intención de este trabajo, persistir y aportar a la dimensión ontológica del debate desde una perspectiva realista y relacional de la empresa.

\section{Lo semántico}

Según Adelstein (2013), a principios del siglo XX, Maitland (1911) ya había afirmado que el sentido común colectivo le atribuía a la empresa una responsabilidad en la sociedad y consideraba que había que investigar y darle fundamento teórico sólido al hecho de considerar a las corporaciones moralmente responsables. El influyente jurista británico afirmaba que la corporación es una realidad fenoménica que la gente trata como persona moral, bajo un sentimiento moral común que surge de la experiencia (Adelstein, 2013). Este uso del lenguaje es un hecho que varios autores (McMahon, 1995; Moore,1999; Blair, 2015) siguen citando como fundamento relevante ya que consideran que refleja la expectativa moral de la sociedad sobre el comportamiento de las empresas.

McMahon (1995) afirma que el lenguaje puede tener un uso práctico o fecundo. Por ejemplo, cuando la ley señala que la empresa es una persona, lo hace para facilitar la actividad económica y no para hacer real a la empresa, que es solo una ficción jurídica. Velasquez (2003) analiza en detalle esta cuestión del uso común del lenguaje en la atribución de responsabilidad moral a la empresa y concluye que el lenguaje metafórico, como tal, no tiene fundamento in re. Por ejemplo, es posible decir "el movimiento del trompo", lo que no implica que el trompo sea causa de su movimiento. Del mismo modo, es posible decir "la empresa contamina", sin embargo, la contaminación es causada por los miembros individuales de 
la empresa. Velásquez afirma de forma contundente que atribuir intenciones a la empresa cuando no es posible predicar la intención a sus miembros individuales (por ejemplo, realizar un contrato), no implica que un grupo organizado sea realmente un agente intencional. Es decir, en este tipo de atribución el lenguaje se usa "como si" y no en sentido literal. El lenguaje describe las acciones de la empresa por analogía o metafóricamente, como si tuviese intenciones reales. Velásquez afirma que el lenguaje metafórico es pragmático y no realista y por tanto de la atribución metafórica no se puede pasar a lo real.

Walt \& Schwartzman (2017), por su parte identifican el análisis ontológico de las corporaciones con meras descripciones innecesarias e insuficientes para determinar si la corporación tiene derechos o deberes. Para estos autores, la AME solo hay que analizarla o bien semánticamente, o bien moralmente. Y la cuestión semántica trata acerca de si esos derechos se atribuyen de modo no-metafórico.

Este tipo de enfoque se encuadra claramente en una perspectiva nominalista en la que el lenguaje sostiene una relación superficial con la realidad denotada, una relación arbitraria o pragmática, sin el fundamento de la adecuación del intelecto a la cosa (ver en Tomás de Aquino en adaequatio intellectus et rei). Es decir, ni el lenguaje ni el intelecto alcanzan a conocer la naturaleza de la realidad. Esta perspectiva semántica del debate no acaba de dar cuenta de la compleja realidad social de la empresa y escapa a la riqueza y profundidad de la predicación análoga ${ }^{11}$.

\section{LO RELACIONAL}

En busca de una posición intermedia en el debate de AME, superadora del individualismo y el colectivismo, algunos autores subrayan la importancia de la trama de vinculaciones e interacciones que se dan en la empresa. Seguiremos en este epígrafe los argumentos de Gibson (2011) y Adelstein (2013) en tanto autores ilustrativos de la búsqueda de una respuesta intermedia. Ambos proponen variaciones sobre la propuesta de French en torno a lo relacional.

11 La analogía también dice relación a la realidad metafísica y no es una mera cuestión de cómo se piensan las esencias. La analogía suele permitir al intelecto ganar en precisión. (García López, 1974: 203). 


\subsection{Kevin W. Gibson}

Gibson es profesor de ética empresarial en Marquette University, EE. UU. En su profundo análisis, este autor afirma que existe una "falsa dicotomía entre la responsabilidad moral individual o de la firma". El debate sobre AME debe focalizarse en la "esencial naturaleza intersubjetiva" de la acción moral en una organización, es decir, "tiene que discernirse al nivel de las interacciones bidireccionales entre individuo y corporación" (Gibson, 2011: 71). Para el autor, lo esencial indagar "si trabajar en un entorno corporativo puede influenciar a los individuos para el bien o para el mal" (Gibson, 2011: 72). Se ha de profundizar dónde la cultura organizacional da forma, modela y restringe el modo en que los líderes y los gerentes toman sus decisiones éticas.

Las corporaciones moldean a las personas y las personas moldean a la organización en una continua negociación el intercambio recíproco de valores. En este intercambio, lo crítico es la autoridad y el rol. Gibson (2011) destaca que el rol de gerente acarrea los valores organizacionales y se espera que el comportamiento del individuo en ese rol los refleje. De acuerdo con la teoría de la agencia (Jensen \& Meckling, 1976), los gerentes de la empresa son agentes o representantes de los intereses de los accionistas. En esa relación, el agente accede a más información que el principal (accionista), por tanto, existe un riesgo moral (moral hazard) de que el agente no actúe representando los intereses del principal e incumpla su obligación fiduciaria (fiduciary duty). Siguiendo a French, Gibson (2011) afirma que entre los altos directivos y los accionistas se da una "agencia por afinidad" (affine agency) donde sus intereses están más atados a los de su empleador. La afinidad, según Gibson, hace que el empleado se sienta satisfecho no solo cuando cumple sus objetivos, sino cuando también lo hace el empleador.

"Hacer de la empresa un actor moral y legal contribuye a reconocer que el tono moral de la empresa altera y refleja el comportamiento de sus miembros" (Gibson, 2011: 72). Por tanto, la responsabilidad corporativa se ubica "en puntos subjetivos y en puntos intersubjetivos de decisión". Ahora bien, reconocer la influencia de las comprensiones compartidas ${ }^{12}$,no implica de ningún modo "una abdicación de la responsabilidad personal en favor de la firma", ni tampoco excusar al individuo culpable (Gibson, 2011: 73).

Para Gibson, la empresa tiene un estatus metafísico distinto al de los individuos, pero no es una persona moral. Si bien no señala qué tipo de 12 Gibson señala que French se refirió a la presencia en la empresa de shared
understandings en las que se manifiesta la intencionalidad de la empresa. 
estatus metafísico tiene, afirma que, dado que los valores institucionales cambian a las personas, esto hace que "la corporación no sea tan externa" a sus integrantes. (Gibson, 2011: 78).

\subsection{Richard P. Adelstein}

Adelstein es profesor en Wesleyan University, EE. UU. En su extenso artículo (Adelstein, 2013) ofrece un análisis de la AME de tipo kantiano. $\mathrm{Si}$ bien reconoce que empíricamente se dan hechos sociales e institucionales y que la empresa es uno de ellos, considera que estos son determinados por las acciones e interacciones de los individuos. A esta posición la denomina "individualismo razonable". Adelstein define la empresa bajo la noción de relacionalidad y las categorías kantianas de tiempo y espacio.

En cuanto a su dimensión temporal, la empresa es un conjunto de contratos relacionales ongoing o bien in performance (que se están ejecutando) que rigen las relaciones a largo plazo que los participantes de la empresa esperan preservar. Lo que Adelstein llama "contrato relacional constitutivo de la firma" es una recreación de la ECDI de French ${ }^{13}$ y consiste en un sistema de roles, relaciones y procedimientos a través del cual la firma es gobernada y operada.

En cuanto a la dimensión espacial de la empresa, lo que constituye a la firma como tal es el acuerdo de sus participantes, es decir, la empresa se convierte en un contrato relacional cuando la gente actúa en tiempo real en sus roles y relaciones definidas por el contrato. Allí la empresa adquiere presencia (physicality) ${ }^{14}$ (Adelstein, 2013: 164).

Cuando se ve que los individuos actúan de acuerdo con dicho contrato, el resultado de sus interacciones se convierte en un hecho institucional: las acciones de la firma. Tanto sus integrantes como los de fuera reconocen en la empresa una "unidad social activa". "Los otros reconocen que yo estoy haciendo algo solamente como parte de nuestro hacer algo". (Adelstein, 2013: 163).

De esas interacciones entre individuos guiadas por el contrato (roles, relaciones definidas) "emergen rutinas comportamentales e idiosincráticas", emergen patrones y un state of mind o intencionalidad colectiva donde "un grupo de individuos comparte una conciencia del nosotros (we consciousness)". De sus rutinas e interacciones emergen las capacidades organizacionales que "son productos irreductibles de la misma." Estas capacidades son poderes reales de percepción, cognición, deliberación y acción

13 Adelstein (2013: 169) se distingue a sí mismo de French diciendo que su enfoque es positivo o descriptivo y el de French es normativo.

14 Propongo traducir physicality en el contexto del artículo, como "presencia". 
para lograr sus objetivos que superan a los de las personas individuales (Adelstein, 2013: 171)

En cuanto a su estatus moral, los actos-con-propósito de las empresas son consecuencia de la interacción de los individuos y son irreductibles a los actos-con-propósito de los individuos. Por tanto, hay una "responsabilidad residual" que solo puede ser atribuida a la firma (Adelstein, 2013: 167).

En cuanto a su estatus metafísico, las empresas son más que cosas, pero menos que personas (Adelstein, 2013: 173). Las personas vivas, en sentido kantiano, son fines en sí mismos y no medios. En cambio, las empresas son "máquinas inteligentes", son instrumentos de fines humanos ${ }^{15}$. Adelstein, en coherencia con su trasfondo kantiano, afirma que las empresas "son, en esencia, ideas u objetos mentales". "Que sea una empresa la que está haciendo algo es verdad, solamente, debido a lo que la gente piensa" (Adelstein, 2013: 164).

En síntesis, para ambos autores, Gibson y Adelstein, la empresa no es una persona moral, pero tampoco es reductible a los individuos ni a sus actos. Para ambos, la empresa se caracteriza por la relacionalidad que se realiza un ordenamiento (la ECDI o el contrato relacional) que organiza a sus integrantes. Mientras que para Adelstein, la existencia de la empresa en sí es un fenómeno empírico-social instantáneo que en "esencia" es tan solo un objeto mental, para Gibson la realidad de la empresa es "no tan externa a la del individuo". Es decir, podría inferirse que, para ambos autores, aunque de diferentes modos, la empresa tendría una especie de existencia intencional, esto es en lenguaje tomista, en el conocimiento o en la mente del sujeto.

\section{EL INDIVIDUO Y EL TODO}

La respuesta aristotélico-tomista que aquí se ofrece para la cuestión ontológica de la empresa (EOE) se basa en un trabajo previo y detallado (Preziosa, 2016 ${ }^{16}$ ) en el que se aplica a la empresa el análisis del grupo social realizado por el filósofo iusnaturalista argentino Julio Guido Soaje Ramos

15 Las empresas no son personas, pueden poseer otras empresas, pero no pueden poseerse a sí mismas ni tienen derecho a la vida. Por ejemplo, si la empresa es vencida por una competencia justa y no da ganancias, no tiene derecho a seguir viviendo como si lo tiene una persona viva.

16 En Preziosa (2016) y en este escrito se cita la paginación del mimeo inédito (Soaje, 1969) que apareció publicado de forma completa con posterioridad (Soaje, 2014, 2015 y 2016-2017). 
(1918-2005). Soaje fue profesor de ética y filosofía del derecho en la Universidad Católica Argentina y en la Universidad Católica de Valparaíso entre otras prestigiosas universidades de Latinoamérica.

Soaje se pregunta ¿en qué consiste la realidad del grupo?, ¿se agota en la realidad de los miembros?, o chay algo real que sea propio del grupo en cuanto tal? (1969: 11-15). Según este autor, para realizar una completa y acabada caracterización metafísica del grupo social es necesario explicar primero cómo y dónde se da su unidad.

En cuanto al "cómo", Soaje distingue la unidad objetiva y la subjetiva. La unidad objetiva es la que resulta del fin u objeto que motiva a los integrantes del grupo, por ejemplo, fabricar un nuevo producto. Ahora bien, que varias personas trabajen en lo mismo no implica que constituyan un grupo. Para que haya grupo social tiene que haber sinergia y articulación de esfuerzos donde cada uno cumpla una acción parcial. La unidad subjetiva, entonces, es la acción total y social que resulta de la articulación de las acciones individuales. (Soaje, 1969: 14). La ECDI de French (1979) y el contrato relacional de Adelstein (2013), expresados en términos de Soaje, constituirían la articulación que otorga unidad subjetiva a la empresa.

En cuanto a "dónde" se da la unidad, Soaje afirma que la empresa no existe "en sí", sino que existe "en otro", existe en sus integrantes. En términos aristotélicos, no todas las realidades son del mismo tenor. Aristóteles identifica diez categorías del ser, de las cuales nueve son accidentes. Los accidentes existen "en otro" y la substancia existe en sí. Por ejemplo, el color de una manzana no existe por sí solo, sino que existe en la manzana. Las acciones de una persona tampoco existen en sí, por ejemplo, la libertad de Pedro existe solo en Pedro. Sin embargo, que exista "en otro", no la convierte en una realidad menos importante. El grupo de amigos de Pedro existe en sus integrantes, no existe en sí. Sin embargo, la realidad del grupo de amigos no es menos real que las personas que la integran, sino tan solo una realidad más frágil.

La empresa donde Pedro trabaja conforma un "todo", pero es un todo accidental y no sustancial. En parte, la fragilidad del todo accidental es tal porque depende de la libertad de las personas. Sus integrantes pertenecen a la organización con diversos grados de voluntariedad y diversos grados de conciencia. (Soaje, 1969: 8-9). ${ }^{17}$

17 Para Adelstein, el acuerdo de los integrantes al contrato relacional se da en cada momento de forma libre y voluntaria, aunque puedan experimentar algunos grados de inequidad o de coerción (2013: 179). 
Según Soaje (1969) es posible distinguir diversos tipos de acciones libres $\left(\right.$ praxis $\left.^{18}\right)$ de los integrantes de la empresa. En primer lugar, se encuentran las ya mencionadas acciones individuales que constituyen al grupo y componen la sinergia. Estas pueden ser o bien externas y observables, por ejemplo, sus actos comunicacionales, o bien interiores al individuo, por ejemplo, sus prejuicios. En segundo lugar, se encuentran las también ya mencionadas acciones colectivas que resultan de la sinergia y "emanan" del grupo ya constituido. Estas acciones colectivas son, o bien exteriores y "objetivas", por ejemplo, su comportamiento en el mercado, o bien interiores al grupo o "subjetivas", como por ejemplo los valores compartidos en la empresa. A los emergentes de las interacciones de la empresa identificados por Adelstein - tales como la intencionalidad colectiva y la "conciencia del nosotros"- Soaje los ubicaría en las praxis interiores o conductas subjetivas del grupo.

Entonces, la unidad de la empresa tiene origen en algo que se busca realizar en común y que motiva a los integrantes a organizarse y articularse sinérgicamente. La acción colectiva que emana de la sinergia remite a "algo más que, en rigor, concierne al grupo formalmente considerado". Ese algo más, no reductible a las acciones individuales, es un todo accidental, un todo práctico (que se funda ónticamente en las praxis) y un todo análogo ${ }^{19}$. (Soaje, 1969: 48). Asimismo, la unidad de la empresa radica tanto en las conductas exteriores como en las conductas interiores de sus integrantes. Tanto en las acciones de producción, comercialización, comunicación, como en su cultura organizacional, sus valores y creencias compartidas.

\section{LA RELACIÓN}

La teoría de la relación aristotélico-tomista es el núcleo de esta respuesta a la cuestión EOE. Soaje señala que la tradición escolástica siempre ha considerado al accidente relación como la realidad más tenue de todas las categorizadas por Aristóteles. La relación es una realidad "en parte enigmática", "mínima y poco firme".

Lo que la diferencia de otros accidentes es que ordena al ente hacia otro y que necesita de un fundamento. En cuanto a que la relación ordena al ente hacia otro, Soaje (1969) explica que, si bien "todo accidente informa a su sujeto de inherencia”, lo especifico de la relación es que se agota en

18 Soaje utiliza el vocablo praxis y con ello se refiere al actuar humano concreto, lo utiliza como sinónimo de acción y opuesto a contemplación. No utiliza el vocablo "praxis" según la tradición que la contrapone a póiesis (crear o fabricar).

19 "La analogía permite acceder a la abstracción de un todo, que llama potestativo, que no es ni la esencia (el todo universal abstracto), ni un todo integral que se pueden subdividir en partes como un cuerpo viviente o una máquina” (García López, 1974: 214). 
"ser con respecto a otro". En cuanto a la necesidad de fundamento, Soaje explica que la relación se compone de cuatro elementos: el sujeto, el término, la relación misma y el fundamento de la relación. Por ejemplo, Pablo es padre de Santiago, por tanto, tienen una relación de paternidad. Pablo es el sujeto y Santiago el término de la relación de paternidad. El fundamento de la relación, que se define como "la razón por la cual el sujeto se refiere al término", en este caso es el acto biológico de generación ${ }^{20}$.

Identificando sus elementos se pueden distinguir las relaciones reales de las relaciones de razón. En la relación real, el sujeto de la relación es modificado por ella. En cambio, en la relación de razón, nada cambia. Por ejemplo, cuando nace Santiago y Pablo se convierte en padre, Pablo adquiere una nueva cualidad, un nuevo modo de ser a causa de esa relación. Pablo resulta modificado por la relación, ahora es padre. Por tanto, la paternidad es una relación real. En cambio, cuando se dice, por ejemplo, que un kilogramo de plumas es igual a un kilogramo de pan, la relación de igualdad es una consideración de la mente que no modifica ni las plumas ni el pan. Por tanto, la igualdad es una relación de razón. En la relación real, el fundamento es real; en la relación de razón, el fundamento es una "consideración de la mente" y no pone nada real en el sujeto ${ }^{21}$.

Este es el fundamento central: "Cuando se discute si el grupo tiene o no realidad, o si toda la realidad social consiste en la de las personas, todo el problema radica en si hay o no relaciones reales" (Soaje, 1969: 15).

Soaje identifica en el grupo social tres tipos de relaciones reales. (a) Las relaciones de semejanza que se fundan en el accidente aristotélico de la cualidad y son las menos importantes porque en ellas no radica la unidad del grupo, aunque la pueden favorecer. Por ejemplo, tener la misma profesión. (b) Las relaciones de interacción o de acción reciproca que se basan en el accidente aristotélico de acción-pasión y Soaje la ejemplifica con la siembra de un campo. (c) Las relaciones de adecuación o de "mutua medición ${ }^{22 " ~ p a r a ~ l a ~ q u e ~ S o a j e ~ b r i n d a ~ t r e s ~ e j e m p l o s: ~ l a ~ r e l a c i o ́ n ~ d e ~ c o n o c i-~}$ miento (por la que el intelecto se adecua a la cosa), la relación de mando y obediencia (por la que el subordinado adecua su conducta al mandato de su superior) y la relación entre la idea ejemplar (que, por ejemplo, tiene el escultor en mente) y lo que se realiza a la manera de esa idea ejemplar (los golpes del cincel que buscan plasmar la idea en mente).

20 Si hablásemos de una relación de filiación, Santiago sería el sujeto y Pablo, el término. El fundamento sería el mismo.

21 En las relaciones de razón, alguno de los cuatro elementos solamente está en la mente de quien predica la relación.

22 Las relaciones de adecuación son presentadas por Soaje con esta expresión en latín: "secundum commensurationem esse et veritatem". Se propone traducirla como: "según la mutua medición del ser y la verdad". 


\section{LA EMPRESA COMO ENTE REAL}

"Si todas las relaciones son de razón, el grupo formalmente en cuanto grupo no tendría una realidad distinta de la de los miembros" (Soaje, 1969: 15). Podría afirmarse que tanto para Velásquez como para Adelstein, la realidad de la empresa se reduce a una consideración de la razón. Velásquez considera que las interacciones intra-empresa y sus resultados no reductibles a los individuos no alcanzan para "reificar" el grupo. Para Adelstein, por su parte, la unidad social observable en las acciones de los integrantes de la empresa es solo un fenómeno empírico de espacio y tiempo y un objeto mental. Soaje coincidiría con Velásquez al decir que de la empresa solo se puede predicar que es una persona, organismo o sustancia por analogía metafórica (Soaje, 1969: 9). Sin embargo, a Soaje esta proposición no lo lleva a negar la realidad grupal social. Soaje, en términos de Velásquez, "reifica" al grupo, le otorga realidad, pero no afirma que sea una realidad sustantiva, sino una realidad poco firme que se funda en las acciones libres de sus integrantes. La empresa existe en sus integrantes, pero no en sentido intencional, sino real ya que se distingue de ellos porque los modifica, generando en ellos nuevos modos de ser accidentales.

Ahora bien, es posible observar en la empresa variados tipos de relaciones de interacción y de adecuación ${ }^{23}$ que permiten dar fundamento a esta realidad, cuya fragilidad no la hace menos real. Las relaciones de interacción surgen de la búsqueda de realizar el objeto en común (por ejemplo, un nuevo emprendimiento) y se dan entre los integrantes de la empresa, entre áreas de la empresa (marketing, producción, finanzas, etc.), entre roles, niveles jerárquicos, etc. Estas interacciones ocurren mediante la comunicación, la negociación, la deliberación, la toma de decisiones y generan compromisos entre las partes y hábitos en quienes interactúan.

Algo más profundas son las relaciones de adecuación, que presuponen las relaciones de interacción. Kenneth Goodpaster (2007), por ejemplo, identifica el fenómeno que denomina "adaptación moral" (moral adjustment) que ocurre entre el empleado y la empresa, donde este o bien se adapta a la cultura de la organización, o bien se va de la empresa si advierte que sus valores no son compatibles. Gibson (2011) afirmaba que mediante un intercambio y negociación de valores las corporaciones moldean a las personas y las personas moldean a la organización. Goodpaster y Gibson ilustran lo que Soaje denomina relaciones de adecuación y que define etimológicamente: "adecuación" significa "hacerse igual", así como el vocablo latino commensuratio significa "mutua medición".

23 Se dejan de lado las relaciones de semejanza, porque como afirma Soaje, solo pueden fomentar la unidad del ente empresa, pero no fundar la unidad. 
Un primer ejemplo de relación de adecuación en la empresa es la adecuación de los comportamientos individuales de sus integrantes a las normas. Las normas comprenden - de modo amplio- las estructuras, roles, estatutos, procesos, protocolos, reglas operativas, códigos formales, etc. Como se señaló al explicar la affine agency de French, el gerente se adecua a su rol y por medio de este a los intereses de los accionistas. En este sentido, los empleados se ajustan a las normas, y a su vez, los directivos las modifican para mejorar el logro de los objetivos. A veces la moral de los directivos parece quedar atrapada en los laberintos burocráticos y las burocracias parecen tener "vida propia" (Jackall, 1983). Es decir, las normas-en sentido amplio- habilitan a los directivos y, a la vez, los restringen (Gibson, 2011).

Esta adecuación moral no se da solo con las normas formales, sino también con las normas informales. La cultura organizacional y la mentalidad compartida acarrean y transmiten reglas, usos y costumbres no escritos a los que el individuo interpreta que debe acomodarse. La mentalidad organizacional mueve con sus valores, explicaciones compartidas acerca de cómo funcionan las cosas en esa organización e incluso con racionalizaciones colectivas (Preziosa, 2016).

Un segundo ejemplo de relación de adecuación se da entre las órdenes de las autoridades de la empresa y los comportamientos de los integrantes. Soaje considera que las órdenes de la autoridad caben también dentro de una consideración amplia de las normas. Esta influencia de los directivos sobre sus subordinados se puede realizar no solo por acción y omisión. En la empresa, no sólo las órdenes directas "miden" los comportamientos, sino también los avales implícitos de la autoridad a ciertas prácticas, la institucionalización o rutinización de procesos, la ejemplaridad del comportamiento directivo, los mecanismos de incentivos establecidos, el diseño de los puestos de trabajo y la discrecionalidad en la asignación de recursos (Preziosa, 2012; 2016).

Como tercer y último ejemplo de relación de adecuación, es posible mencionar la que se da entre la aspiración, el ideal o la visión de la empresa (competir en un nuevo mercado, lanzar un nuevo producto, mejorar la calidad, etc.) y todas las acciones mancomunadas que se realizan para lograrlo.

Por tanto, quien integra la empresa se adecua, se ajusta, se mide mutuamente con las normas formales e informales, las órdenes de las autoridades y la visión que se comparte. Todas estas relaciones de conmensuración o adecuación modifican a los sujetos que integran la empresa y permanecen en ella. La interacción y adecuación, en tanto relaciones reales, modifican a sus sujetos en sus conductas exteriores e interiores, en las acciones individuales y las colectivas. 
Por tanto, se puede afirmar que la empresa existe como un ente distinto de sus integrantes, pero inherente a sus conductas. Los sujetos de estas relaciones reales adquieren un nuevo modo de ser - en sentido accidental- que es propio de la cultura de esa empresa. Es decir, adquieren cualidades (accidente aristotélico) por la cotidianeidad y la repetición. Esas cualidades incluyen hábitos propios de esa empresa que otros pueden reconocer como típicos de esa cultura empresaria.

Los hábitos son disposiciones que las personas adquieren mediante la repetición de actos. Estas disposiciones perfeccionan la naturaleza humana, en el sentido de que la naturaleza adquiere nuevos modos de ser (Rodríguez Luño, 1983). Los hábitos facilitan la toma de decisiones en una cierta dirección, sin anular la libertad. Pertenecer a una empresa por un cierto periodo de tiempo, implica la adquisición de varios hábitos que en ese lugar son valorados. Los hábitos si bien son individuales y radican en un sujeto, tienen una dimensión grupal y compartida. En tanto la persona se adecua a la empresa y desea integrarse, pertenecer, imita y adquiere hábitos del grupo. En la empresa, hábitos son, por ejemplo, formas de trabajar, de comunicarse, formas de resolver problemas; o también, estilos de dirección de personas, aprendizajes grupales, desarrollo de habilidades (skills) o capacidades (capabilities) necesarias para un determinado tipo de negocio. Estos hábitos compartidos componen lo que se denomina el know-how de la empresa y generan una diferencia en sus recursos humanos que el mercado laboral, también percibe como tal. El conjunto de cualidades y hábitos que surgen de las rutinas de interacción y adecuación diferencian una organización respecto de otras compañías en el mercado, conformando su realidad, su identidad ${ }^{24}$, su ser.

\section{LA RELACIÓN Y EL ORDEN}

A fin de completar esta caracterización ontológica de la empresa resta responder esta pregunta: ¿qué tipo de realidad tiene la empresa? Para ello, Soaje propone analizar la noción de orden en su implicación mutua con la noción de la relación. "En la relación real se da un orden, respecto, o habitud, que realmente afecta al sujeto del cual se afirma" (1969: 14).

El concepto relación está etimológicamente emparentado con el latín re-fero que significa "llevo hacia" y con el vocablo español respecto ${ }^{25}$ que, a su vez, deriva del latín re-spicio y que traduce como "miro hacia". De la etimología, Soaje infiere que la relación es una realidad que mira hacia otro,

24 Estas rutinas de interacción incluyen también la mano del management (Goodpaster, 1982).

25 DRAE, Respecto: razón, relación o proporción de algo a otra cosa. 
que lleva hacia otro, que es para otro. Por tanto, afirma, la relación es un orden hacia otro $($ ordo ad). Es decir, la noción de orden es más genérica y previa a la noción de relación (Soaje, 1969: 14).

Así como se distinguieron los elementos de la relación, se identifican los elementos del orden. Soaje lo explica con el ejemplo de una biblioteca. Si muchos libros se relacionan con un orden alfabético queda constituida una biblioteca. Si no hay un principio ordenador común a todos los libros no hay biblioteca, sino solo un montón de libros. Así como el montón de libros conforma "un todo por yuxtaposición", la biblioteca conforma un "todo de orden". Entonces, la noción de orden tiene tres componentes: una base plural de elementos, un principio de orden y una relación de los elementos con dicho principio de orden. Soaje define el orden como la "unidad que resulta de la referencia de los elementos del orden a su principio" (1969: 16).

El orden en la empresa es, para French, la ECDI y para Adelstein, el contrato relacional constitutivo. Para Gibson, la cultura de la compañía y su ethos predominante ordenan de un modo no tan externo al individuo.

Para Soaje, el orden es lo que explica la trama relacional a la que se ajustan sus integrantes porque da fundamento a las interacciones y a la reciprocidad entre los miembros y hace ser al grupo. El orden es el fundamento de la relación. En la empresa el fundamento de su realidad relacional es la estructura organizada en vistas del fin de la empresa. Por ejemplo, una nueva empresa se organiza alrededor de ciertas pautas colectivas que sus directivos conciben en función del producto a realizar entre todos. Actuando en relación a pautas colectivas (normas) se va inviscerando ${ }^{26}$ un orden en las vidas de los miembros de la empresa (Soaje, 1969: 54). Por tanto, el orden surge de las exigencias del fin que mueve al grupo. Cada miembro ocupa un rol o jerarquía que se ha de subordinar (incluso la autoridad ${ }^{27}$ ) al orden que se considera adecuado o proporcionado para llegar al fin. En este sentido, el orden modifica a las personas que se ajustan a él, en sus decisiones y en sus hábitos.

El orden es un entretejido vital (Soaje utiliza el vocablo "plexo") de relaciones, es una malla (Rodríguez Coronel, 2012) que enlaza y radica en las acciones de los miembros del grupo. En la empresa el fundamento de su realidad relacional son las normas en sentido amplio (formales, como

\footnotetext{
26 En DRAE no figura "inviscerar". Diccionario online de portugués- español, "inviscerar": "meter en las entrañas", "arraigar".

27 Dado que el orden se sigue del fin, la autoridad no ha de imponer órdenes arbitrarias sino aquellas que permitan la consecución del objetivo. El orden no deviene de la autoridad, sino que la autoridad deviene del orden (Soaje, 1969: 55).
} 
la estructura; informales, como la mentalidad y las directrices de las autoridades) que articulan la sinergia.

Por tanto, la realidad de la empresa consiste en una relación ordenada o en un orden relacional distinto a sus integrantes. Consiste en una unidad motivada por un fin y unida por un orden. Consiste en un entretejido vital que modifica a sus integrantes con nuevas cualidades conformando una realidad tenue, pero irreductible que depende de la libertad de sus integrantes, que no existe solo en su mente, sino que perdura en sus logros, su cultura y hábitos compartidos.

\section{CONCLUSIONES}

La cuestión de la agencia moral de la empresa (AME) constituye un largo y complejo debate en la literatura angloamericana de ética empresarial. Hoy, en Latinoamérica vuelve a tomar relevancia social a partir de la tendencia legal de penalizar a la empresa como un todo, más allá de la penalidad de los directivos. En estas leyes se reconoce la influencia de la organización en el comportamiento de los individuos. El debate AME, que esencialmente discute si se puede predicar responsabilidad de la empresa en sí, se encuentra imbricado con la cuestión del estatus ontológico de la empresa (EOE), que discute si la empresa es un ente distinto de sus integrantes.

Para responder al debate EOE, en primer lugar, se han presentado argumentos de autores que reconocen fenoménicamente la existencia de la empresa como un hecho social, pero esto no los lleva a afirmar que la empresa tenga una realidad distinta a la de sus integrantes, sino que es solo una consideración de la razón o del pragmatismo del lenguaje sin fundamento in re. En segundo lugar, se han presentado argumentos de autores que hace foco en lo intersubjetivo y relacional, sin embargo, no especifican el estatus metafísico de la empresa, aunque reconocen su irreductibilidad a las acciones de los individuos. En tercer lugar, se ha ofrecido una respuesta basada en la metafísica aristotélico-tomista centrada en la noción del accidente relación.

El eje central de la respuesta de este escrito es la noción de relación real, es decir, de aquellas relaciones que modifican a sus sujetos. La modificación -de orden accidental y no sustancial- consiste en la adquisición de nuevas perfecciones o modos de ser, esto es, cualidades y hábitos. Estas modificaciones que sobrevienen a los integrantes de la empresa son resultado de la rutina de interacciones y adecuaciones que realizan por querer pertenecer a la empresa. Si bien esos nuevos modos de ser radican en cada sujeto integrante, son compartidos en cuanto a su forma, ya que resultan de adecuarse a las normas u ordenamiento que articula sus interacciones 
para lograr el fin que se propone la organización empresarial. Este ordenamiento proviene de la estructura formal, la cultura informal y el estilo de dirección de sus autoridades.

La empresa es presentada como un ente real distinto de sus integrantes, pero que radica en ellos, en sus acciones libres. Es una realidad accidental y no sustancial, pero no por ello menos importante. La libertad individual y la necesidad de un fundamento para la relación hacen de la relacionalidad algo tenue y poco firme, pero no por ello menos real. El fundamento de las interacciones y relaciones de adecuación que da forma al todo, a la unidad, es el orden. El orden une de forma objetiva y subjetiva a sus integrantes. Dicho orden, que resulta de las exigencias del fin que se quiere lograr entre todos, es lo que se entreteje de modo vital en las vidas de los integrantes de la empresa, modificándolas. Por tanto, la empresa existe en sus integrantes, pero no de modo intencional sino de modo real, porque los modifica en habilidades y capacidades compartidas que definen la identidad de la empresa y la distingue de otras.

De este modo se ha ofrecido una respuesta intermedia que, frente a los argumentos individualistas, sigue sosteniendo el peso ontológico del individuo y su libertad, pero reconoce la existencia de algo irreductible propio de la empresa. Frente a los argumentos colectivistas, la respuesta provista no sustancializa a la empresa, no la considera una persona o un organismo, pero afirma su realidad. Frente a los argumentos basados en la noción de relacionalidad, el análisis aristotélico-tomista provee un adecuado fundamento a la relación, quitándola del lugar de categoría mental, para ubicarla en una categoría de ser accidental.

Si bien la realidad empírica de la empresa es sumamente compleja, el análisis realizado aborda lo ontológico, la naturaleza relacional y ordenada de una organización empresarial. Por tanto, este análisis puede subyacer a distintas formas jurídicas y económicas de organización empresarial.

\section{REFERENCIAS}

Adelstein, R. (2013). Firms as Persons. Cabiers d'Economie Politique, (65), 161-182. Blair, M. M. (2015). On Corporations, Courts, Personhood, and Morality. Business Ethics Quarterly, 25(4), 415-431.

Buddeberg, E. \& Hecker, A. (2018). Justification Incorporated: A Discursive Approach to Corporate Responsibility. Ethical Theory and Moral Practice, (Preprints), 1-11.

Enderle, G. (1993). What is Business Ethics? En T.W. Dunfee \& Y. Nagayasu (Eds.), Business Ethics: Japan and the Global Economy. Issues in Business Ethics, (5) 33-150. Netherlands: Springer, Dordrecht. 
French, P. A. (1979). The Corporation as a Moral Person. American Philosophical Quarterly, 16, 207-215.

French, P. (1984). The Principle of Responsive Adjustment in Corporate Moral Responsibility: The Crash on Mount Erebus. Journal of Business Ethics, 3(2), 101-111.

García López, J. (1974). La analogía en general. Anuario filosófico, 7(1), 192-223.

Gibson, K. (2011). Toward an Intermediate Position on Corporate Moral Personhood. Journal of Business Ethics, 101 (Supplement 1), 71-81.

Goodpaster, K. E. \& Matthews, J. J. B. (1982). Can a Corporation Have a Conscience? Harvard Business Review, 60(1), 132-141.

Goodpaster, K. E. (2007). Conscience and Corporate Culture. New York: WileyBlackwell Publishing.

Heersmink, R. (2017). Distributed Cognition and Distributed Morality: Agency, Artifacts and Systems. Science and Engineering Ethics, 23(2), 431-448.

Jackall, R. (1983). Moral Mazes: Bureaucracy and Managerial Work. Harvard Business Review, 61(5), 118-130.

Jensen, M. C. \& Meckling, W. H. (1976). Theory of the Firm: Managerial Behavior, Agency Costs and Ownership Structure. Journal of Financial Economics, 3(4), 305-360.

McMahon, C. (1995). The Ontological and Moral Status of Organizations. Business Ethics Quarterly, 5(3), 541-554.

Moore, G. (1999). Corporate Moral Agency: Review and Implications. Journal of Business Ethics, 21(4), 329-343.

Phillips, M. J. (1995). Corporate Moral Responsibility: When it Might Matter. Business Ethics Quarterly, 5(3), 555-576.

Preziosa, M. M. (2006). Agencia moral de la empresa: el aporte del análisis causal aristotélico. Journal of Economics, Finance and Administrative Science (Ex-Cuadernos de Difusión. Universidad ESAN), 11(21), 9-24.

Preziosa, M. M. (2012). Líderes que no obligan al heroísmo. Revista Cultura Económica, 30(84), 46-50.

Preziosa, M. M. (2016). La mentalidad compartida en la empresa. Buenos Aires: Editorial Teseo.

Rodríguez Coronel, M. M. (2012). A vueltas con agencia moral: una perspectiva crítica. Quaderns de Filosofía i Ciencia, 42, 127-137.

Rodríguez Luño, A. (1983). La virtud moral como hábito electivo según Santo Tomás de Aquino. Persona y derecho: Revista de fundamentación de las Instituciones Jurídicas y de Derechos Humanos, (10), 209-234.

Soaje, G. (1969). El grupo social. Mimeo inédito. Cátedra Filosofía Social, Facultad de Filosofía y Letras, Pontificia Universidad Católica Argentina.

Soaje, G. (2014). El grupo social (1 parte). Ethos: Revista de Filosofía Práctica, (29). Soaje, G. (2015). El grupo social (2० parte). Ethos: Revista de Filosofía Práctica, (30). Soaje, G. (2016-2017). El grupo social (3º parte). Ethos: Revista de Filosofía Práctica, (31-32).

Soares, C. (2003). Corporate Versus Individual Moral Responsibility. Journal of Business Ethics, 46(2), 143-150. 
Velasquez, M. (2003). Debunking Corporate Moral Responsibility. Business Ethics Quarterly, 13(4), 531-562.

Walt, S. \& Schwartzman, M. (2017). Morality, Ontology, and Corporate Rights. Law \& Ethics of Human Rights, 11(1), 1-29.

Sumario: 1. Introducción; 1.1. La perspectiva legal; 1.2. La cuestión del estatus moral de la empresa en la disciplina ética empresarial; 1.3. La cuestión del estatus ontológico de la empresa; 2. Lo colectivo y lo individual; 2.1. Peter A. French; 2.2. Manuel G. Velasquez; 3. Lo ontológico y lo moral; 4. Lo relacional; 4.1. Kevin W. Gibson; 4.2. Richard P. Adelstein; 5. El individuo y el todo; 6. La relación; 7. La empresa como ente real; 8. La relación y el orden; Conclusiones; Referencias. 